\title{
Herpes Simplex Virus Capsid Maturation Visualized by Time-Lapsed Cryo-EM
}

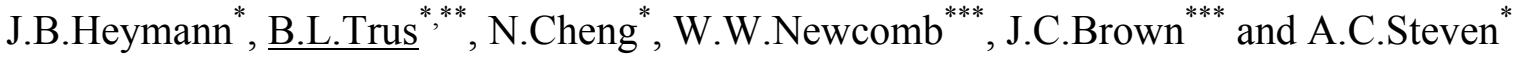 \\ * LSBR, NIAMS, and ** CBEL, CIT, NIH, Bethesda, Maryland 20892 \\ *** Department of Microbiology, University of Virginia Health System, Charlottesville, VA 22908
}

The 192 MDa capsid of herpes simplex virus (HSV) is assembled in the host nucleus from three structural proteins, aided by a scaffolding protein and a maturational protease. The initial particle is a procapsid, with the major capsid protein, VP5, forming capsomers held together by triplexes, triangular structures composed of the other two proteins, VP19C and VP23, and surrounding a core of scaffolding protein and protease. During maturation, the protease is activated and interactions between capsomers are established, leading to a change in shape and stabilization of the capsid. In vivo, the scaffolding protein and protease inside the capsid are replaced by viral DNA.

Procapsids were isolated from BHK cells infected with the HSV tsProt.A mutant strain [1]. In PBS at $20^{\circ} \mathrm{C}$, procapsids mature spontaneously over several days [2]. Preparations were sampled for cryoEM at time points 0 (directly after purification), $24 \mathrm{hr}, 48 \mathrm{hr}$ and $96 \mathrm{hr}$. Our approach to identifying transition intermediates was based on image classification. In all, 5000 particles from 64 focal pairs of micrographs at the 4 time-points were extracted, CTF-corrected, and analyzed. We then generated 19 maps as incrementally weighted linear combinations of pre-existing maps of the procapsid and the mature A-capsid [2]. Particle orientations were determined with respect to each map with a variant of the PFT algorithm [3]. Each particle was assigned to a reference map on the basis of correlation coefficients, with about $40 \%$ rejected as correlating poorly with all maps. The reference models were revised at each cycle and eventually converged to a solution at a resolution of about 25 Å representing a consistent time-ordered sequence of 17 maps of the maturation process.

In the procapsid, VP5 forms hexons and pentons that interact only indirectly via the triplexes [4] (Fig. 1). Procapsid hexons are rings of VP5 perturbed from 6-fold symmetry in ways that depend on the location in the surface lattice. The external protrusions of the E-hexon and P-hexon are oval, while the $\mathrm{C}$-hexon is triangular. In the mature capsid (Fig. 1), the hexons are almost circular with a pseudo-6-fold local symmetry. During maturation, the procapsid changes from a spherical to a polyhedral shape, with little change in diameter (Fig. 1). All major changes coincide with this angularization of the capsid. On the inner surface, the first connections form along the line from the 5-fold to the 2-fold axes, i.e., involving the pentons, P-hexons and E-hexons (Fig. 1). Shortly after, all connections to the C-hexons are established, forming a tightly interlocked capsid floor. Simultaneously, the hexon protrusions regularize, rotating by about $15^{\circ}$ and condensing into closely packed rings. The 17 maps gave few indications of structurally distinct intermediates: most were procapsid-like or mature capsid-like. Our series of 17 maps is time ordered as indicated by the classification of the particle images at each time point, progressing from more procapsid-like particles to more mature capsid-like particles. However, these maps do not represent equal time intervals. The main transition involves maps 7-10 (Fig. 1) and we conclude that when maturation is triggered for a given particle, it takes place abruptly and cooperatively. Maturation appears to have its basis in relative rotational changes of domains of VP5. 

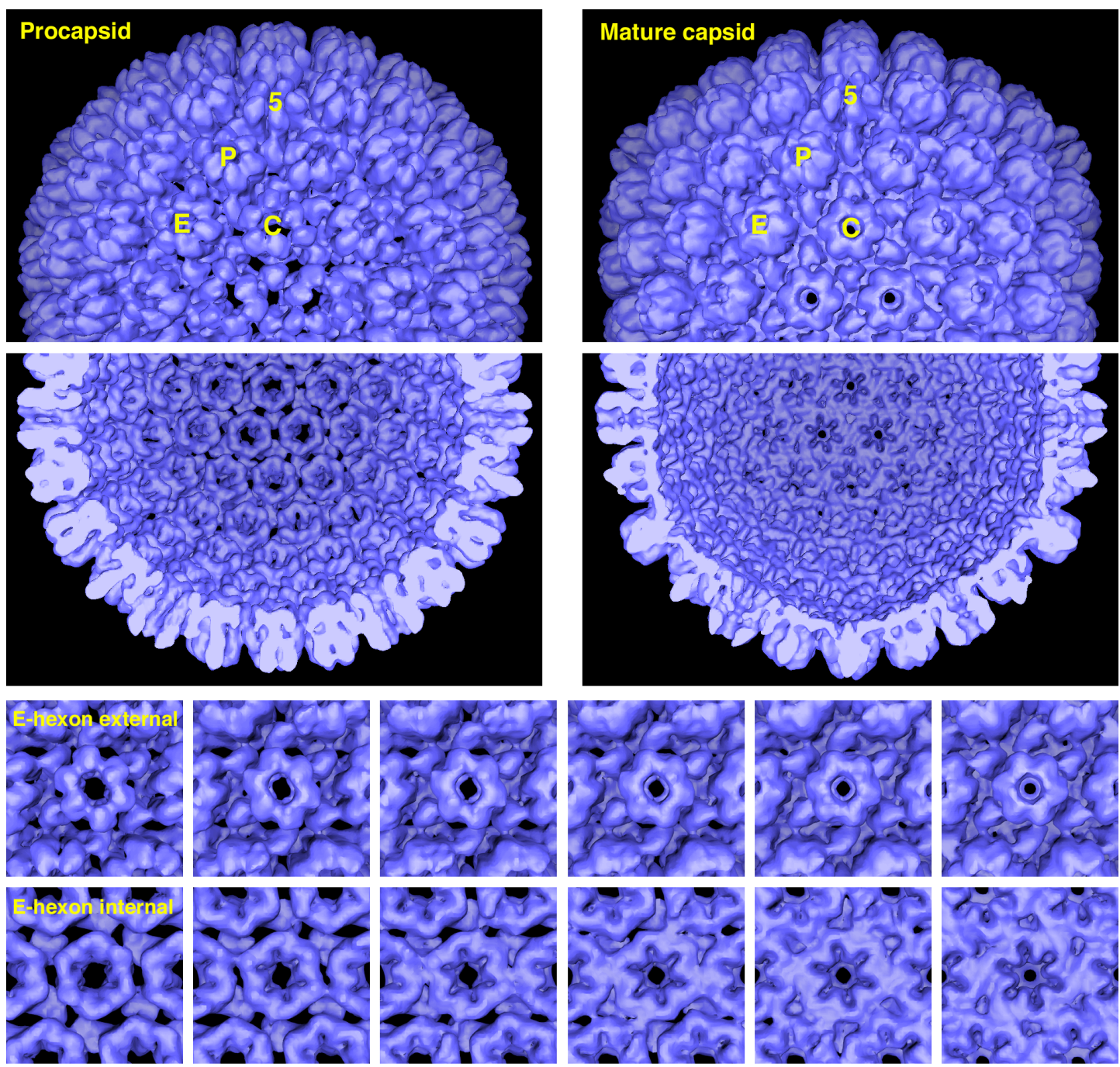

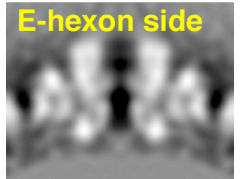

Map 1

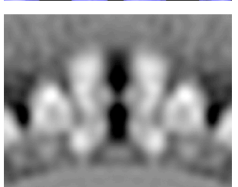

Map 7

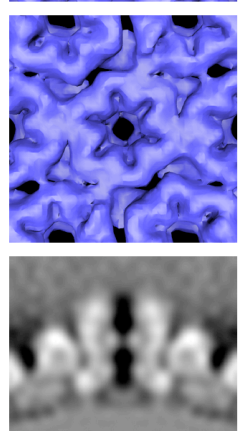

Map 9
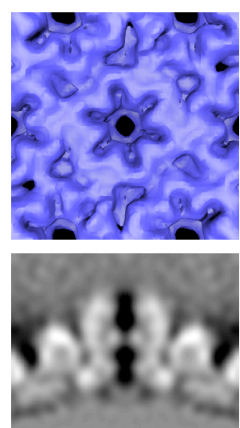

Map 10

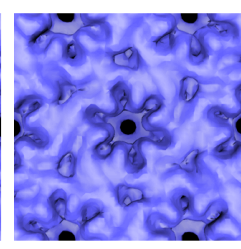

Map 8

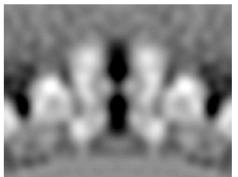

Figure 1: Maturation of the HSV capsid, showing the procapsid (top left), and the mature capsid (top right) viewed down the three-fold axis from the outside and the inside. The bottom three rows show the transformation of the E-hexon, with major changes observed over the central four maps of the 17 map series. The hexons are well separated from each other in the procapsid, only connected by the triplexes (internal views). Connections form between the E-hexons and P-hexons to the side before connections to the C-hexons above and below. In the side views (bottom), these connections appear below the triplexes (arrow). 5: penton; P: peripentonal hexon; E: edge hexon; C: central hexon.

\section{References}

[1] M. Gao et al. (1994) J. Virol. 68, 3702-12.

[2] W. W. Newcomb et al. (2000) J. Virol. 74, 1663-73.

[3] T. S. Baker and R. H. Cheng (1996) J. Struct. Biol. 116, 120-130.

[4] B. L. Trus et al. (1996) J. Molec. Biol. 263, 447-462. 